University of Nebraska - Lincoln

DigitalCommons@University of Nebraska - Lincoln

USDA National Wildlife Research Center - Staff Publications
U.S. Department of Agriculture: Animal and Plant Health Inspection Service

2015

\title{
Mechanisms of antimicrobial resistant Salmonella enterica transmission associated with starling-livestock interactions
}

James C. Carlson

USDA/APHIS/WS National Wildlife Research Center, james.c.carlson@aphis.usda.gov

Doreene R. Hyatt

Colorado State University

Jeremy W. Ellis

USDA/APHIS/WS National Wildlife Research Center

David R. Pipkin

U.S. Department of Agriculture, Animal and Plant Health Inspection Service, Texas Wildlife Services

Anna M. Mangan

USDA/APHIS/WS National Wildlife Research Center

See next page for additional authors

Follow this and additional works at: https://digitalcommons.unl.edu/icwdm_usdanwrc

Part of the Life Sciences Commons

Carlson, James C.; Hyatt, Doreene R.; Ellis, Jeremy W.; Pipkin, David R.; Mangan, Anna M.; Russell, Michael; Bolte, Denise S.; Engeman, Richard M.; DeLiberto, Thomas J.; and Linz, George M., "Mechanisms of antimicrobial resistant Salmonella enterica transmission associated with starling-livestock interactions" (2015). USDA National Wildlife Research Center - Staff Publications. 1737.

https://digitalcommons.unl.edu/icwdm_usdanwrc/1737

This Article is brought to you for free and open access by the U.S. Department of Agriculture: Animal and Plant Health Inspection Service at DigitalCommons@University of Nebraska - Lincoln. It has been accepted for inclusion in USDA National Wildlife Research Center - Staff Publications by an authorized administrator of DigitalCommons@University of Nebraska - Lincoln. 
Authors

James C. Carlson, Doreene R. Hyatt, Jeremy W. Ellis, David R. Pipkin, Anna M. Mangan, Michael Russell, Denise S. Bolte, Richard M. Engeman, Thomas J. DeLiberto, and George M. Linz 


\title{
Mechanisms of antimicrobial resistant Salmonella enterica transmission associated with starling-livestock interactions
}

\author{
James C. Carlson ${ }^{\mathrm{a}, *}$, Doreene R. Hyatt ${ }^{\mathrm{b}}$, Jeremy W. Ellis ${ }^{\mathrm{a}}$, David R. Pipkin ${ }^{\mathrm{c}}$, \\ Anna M. Mangan ${ }^{a}$, Michael Russell ${ }^{\mathrm{b}}$, Denise S. Bolte ${ }^{\mathrm{b}}$, Richard M. Engeman ${ }^{\mathrm{a}}$, \\ Thomas J. DeLiberto ${ }^{\mathrm{a}}$, George M. Linz ${ }^{\mathrm{d}}$ \\ ${ }^{a}$ U.S. Department of Agriculture, Animal and Plant Health Inspection Service, Wildlife Services, National Wildlife Research Center, \\ 4101 LaPorte Avenue, Fort Collins, CO 80521, USA \\ ${ }^{\mathrm{b}}$ Colorado State University, Veterinary Diagnostic Laboratories, College of Veterinary Medicine and Biomedical Science, 1644 Campus \\ Delivery, Fort Collins, CO 80523-1644, USA \\ ${ }^{\mathrm{c}}$ U.S. Department of Agriculture, Animal and Plant Health Inspection Service, Texas Wildlife Services, P.O. Box 690170, San Antonio, TX \\ 78269, USA \\ ${ }^{\mathrm{d}}$ U.S. Department of Agriculture, Animal and Plant Health Inspection Service, Wildlife Services, National Wildlife Research Center, \\ 2110 Miriam Circle, Suite B, Bismarck, ND 58501-2502, USA
}

\section{A R T I C L E I N F O}

\section{Article history:}

Received 11 December 2014

Received in revised form 6 March 2015

Accepted 12 April 2015

\section{Keywords}

Antimicrobial resistance

European starlings

Bacteria

Enterobacteriaceae

Salmonella enterica

\begin{abstract}
A B S T R A C T
Bird-livestock interactions have been implicated as potential sources for bacteria within concentrated animal feeding operations (CAFO). European starlings (Sturnus vulgaris) in particular are known to contaminate cattle feed and water with Salmonella enterica through their fecal waste. We propose that fecal waste is not the only mechanisms through which starlings introduce $S$. enterica to CAFO. The goal of this study was to assess if starlings can mechanically move $S$. enterica. We define mechanical movement as the transportation of media containing S. enterica, on the exterior of starlings within CAFO. We collected 100 starlings and obtained external wash and gastrointestinal tract (GI) samples. We also collected 100 samples from animal pens. Within each pen we collected one cattle fecal, feed, and water trough sample. Isolates from all S. enterica positive samples were subjected to antimicrobial susceptibility testing. All sample types, including $17 \%$ of external starling wash samples, contained S. enterica. All sample types had at least one antimicrobial resistant (AMR) isolate and starling GI samples harbored multidrug resistant S. enterica. The serotypes isolated from the starling external wash samples were all found in the farm environment and $11.8 \%(2 / 17)$ of isolates from positive starling external wash samples were resistant to at least one class of antibiotics. This study provides evidence of a potential mechanism of wildlife introduced microbial contamination in CAFO. Mechanical movement of microbiological hazards, by starlings, should be considered a potential source of bacteria that is of concern to veterinary, environmental and public health.
\end{abstract}

Published by Elsevier B.V.

\section{Introduction}

Domestic cattle (Bos taurus) is a known source of bacteria of concern to public health, including Escherichia

\footnotetext{
* Corresponding author. Tel.: +1 970266 6127; fax: +1970266 6138

E-mail address: James.C.Carlson@aphis.usda.gov (J.C. Carlson).
}

coli 0157:H7, methicillin-resistant Staphylococcus aureus (MRSA), and antimicrobial resistant (AMR) Salmonella enterica (Armstrong et al., 1996; Loo et al., 2007; Brichta-Harhay et al., 2011). Additionally, there is a growing body of literature that suggests cattle may not be the only animals within concentrated animal feeding operations (CAFO) that contribute to the emergence and 
environmental dissemination of antimicrobial resistant (AMR) bacteria. Wildlife incursions into CAFO are known to cause economic damage as a consequence of feed consumption (Shwiff et al., 2012) and many of these species have been documented as carriers of E. coli 0157:H7 and AMR S. enterica (Kirk et al., 2002; Gaukler et al., 2009; Kauffman and LeJeune, 2011).

European starlings (Sturnus vulgaris) in particular are known to cause damage to CAFO through the consumption of livestock feed (Dolbeer et al., 1978; Depenbusch et al., 2011). Consumption of livestock feed by starlings is associated with increased probability of detecting $S$. enterica (Carlson et al., 2011a) and length of exposure to finishing rations is associated with $S$. enterica fecal shedding by cattle (Fedorka-Cray et al., 1998). Reducing starling numbers is associated with reductions in $S$. enterica contamination within cattle feed and water supplies (Carlson et al., 2011b). This information suggests that the ecological interactions between starlings and cattle may result in the transfer of bacteria to feed and water supplies and these interactions may contribute to cattle infections, increased cattle fecal shedding, and environmental dissemination of bacteria that is of concern to public health.

Currently the ecological interactions associated with microbiological contamination of CAFO by wild birds are poorly understood. For example, S. enterica serotypes recovered from starling gastrointestinal (GI) tracts did not correspond well to the serotypes recovered from cattle feed and water troughs, even though presence of starlings were one of the most heavily weighted explanatory variables for $S$. enterica contamination within both feed and water troughs (Carlson et al., 2011a). Published research associated with bird-livestock interactions in CAFO have relied on fecal, cloaca, or GI samples to isolate bacteria from birds (Kirk et al., 2002; Pedersen et al., 2006; Gaukler et al., 2009; Carlson et al., 2011a,b; Cernicchiaro et al., 2012). Based upon our behavioral observations of birds in CAFO we hypothesize that, in addition to bird feces, starlings mechanically move cattle feces, on their feet and feathers to cattle feed and water troughs within CAFO.

The objectives of this study were two-fold: (1) determine if starlings can mechanically move $S$. enterica; (2) characterize the serotypes and antimicrobial resistance phenotypes of $S$. enterica isolates obtained from five different sources within a CAFO (starling GI, external starling wash, cattle feces, feed, and water).

For the purposes of this manuscript we group the five sources into two different categories: fecal samples and environmental samples. Fecal samples consist of fecal waste generated by the animals within the CAFO, cattle feces and starling GI samples. Environmental samples consist of sources we suspect may be associated with $S$. enterica-cattle-starling transmission cycle; feed, water and external starling wash samples. We analyze data within and between these two groups because these categories help us to better understand the animals shedding $S$. enterica, the media being consumed that causes foodborne $S$. enterica infections, and potential mechanisms for $S$. enterica contamination in CAFO that could contribute to cattle infections and increased fecal shedding.

\section{Methods}

\subsection{Study area}

We conducted this study on a CAFO in Moore County, TX, USA. The CAFO produced feeder cattle and had a herd size of approximately 50,000 head housed in 382 pens. No other livestock were present and the CAFO had extremely high visitation rates of starlings ( $\geq 10,000$ starlings/day). Cattle were housed 50-150 individuals per pen and were fed a finishing ration consisting of approximately $75 \%$ steam flaked corn and $25 \%$ corn silage. Antibiotics were used for the treatment of sick animals (tetracycline, $350 \mathrm{mg} /$ head/day). Tylan ( $89 \mathrm{mg} /$ head/day) and Rumensin $(150 \mathrm{mg} /$ head/day) were provided in cattle feed prophylactically and to manage coccidian and increase weight gain. Cattle manure was removed from pens two times per month using front-end loaders and dump trucks. Water troughs were supplied by untreated ground water and cleaned biweekly. Feed troughs were cleaned after pens were emptied and animals were taken to market.

\subsection{Sample collections}

To calculate sampling intensity we used the formula published in Guidelines for the Care and Use of Mammals in Neuroscience and Behavioral Research (NRC, 2003; $n=\log \beta(\log p)$. Where $\beta$ is the probability of committing a Type II error and $p$ represents the proportion of animals in the colony that are not infected. To estimate proportion not infected we used $S$. enterica detection estimates within starlings, cattle feces, cattle feed and cattle water troughs collected from the same CAFO in 2009 (Carlson et al., 2011a) and $p$ was calculated by subtracting the percent positive from 1 , for each media. We set $\beta$ at 0.05 . For example, $3 \%$ of starlings within this CAFO were positive for S. enterica in 2009, thus $n=\log (0.05) / \log (1-0.03)$. Using the equation and assumptions above we estimated that 98 samples would be the minimum sampling intensity necessary to reliably detect $S$. enterica on or in European starlings, 41 from cattle fecal, 36 from cattle feed, and 20 from water trough samples. Thus, we concluded that 100 samples per source ( 500 total samples) would be adequate to detect $S$. enterica on European starlings and to characterize the S. enteric isolated from the media sampled within this CAFO.

A total of 100 pens were sampled. We preselected 100 pens using a map of the CAFO. Pens selected for sampling were uniformly spread throughout the facility by selecting every third pen within pen lanes. One cattle feed, water, and fecal sample were collected per pen. Collection of samples occurred during a 4-day period from 23-26 January 2012. All samples were aseptically collected after starlings arrived on the CAFO (approximately 9:00 am CST) and sample collection stopped when birds returned to roost sites (approximately 4:00 pm CST).

We collected feed samples directly from the troughs 15 min after trucks delivered cattle feed. Collection of 
cattle feed was delayed to allow cattle and starlings time to interact and consume rations. Feed troughs consisted of concrete bunkers attached to the outside of the pens, which ran the length of the pen lanes. Trucks filled feed troughs regularly allowing cattle to eat ad libitum. One 25$\mathrm{g}$ sample of cattle feed was collected from each feed trough. Additionally, we collected 30 reference feed ingredients on the last day of data collection to assess if feed contamination occurred at the trough, during storage, or delivery via trucks. Among the 30 reference rations, we collected 10 samples from the silage pile, 10 samples from stored steamed flaked corn and 10 mixed rations directly from truck prior to feeding. All feed samples were aseptically collected in Whirl-Paks ${ }^{\circledR}$ (NASCO, Fort Atkinson, WI 53538) and samples were immediately placed in electric coolers set to $4{ }^{\circ} \mathrm{C}$.

Water troughs were open, auto-filled water basins. All sampled water troughs were located in pens and none of the water troughs could be accessed by cattle in adjacent pens. We collected $100 \mathrm{~mL}$ of water from troughs within the pens in autoclaved $125 \mathrm{~mL}$ plastic vials. Water was sampled vertically up through the water column from the center of the water basin. All water samples were immediately placed in electric coolers set to $4{ }^{\circ} \mathrm{C}$.

We collected cattle fecal samples from the animal pens immediately after an animal was observed defecating. We intentionally avoided sampling mixed fecal media from the pen floor which allowed us to standardize environmental exposure time among fecal samples and to eliminate cross-contamination from birds or other wildlife. We only collected fecal samples if we could reasonably determine, by visual inspection, that the sample was absent of fecal material originating from other cattle. Tengram samples were scraped from the top of the fecal pat with disposable plastic spoons and stored in sterile WhirlPaks ${ }^{\mathbb{R}}$. All fecal samples were immediately placed in electric coolers set to $4{ }^{\circ} \mathrm{C}$.

We collected 100 starlings from CAFO within cattle pens and pen lanes. All starlings were collected with shotguns and birds were only shot after they landed on the ground. Starling collections followed methods conforming to agency policy as stated in United States Department of Agriculture (USDA), Animal and Plant Health Inspection Service (APHIS), Wildlife Service Directive 2.505 and were approved by the National Wildlife Research Center's (NWRC) Internal Animal Care and Use Committee (NWRC protocol, QA-1919). All carcasses were individually bagged in sterile Whirl-Paks ${ }^{\circledR}$ and stored in electric coolers set to $4{ }^{\circ} \mathrm{C}$ until processing. Processing starlings for laboratory analysis occurred away from the CAFO in Dumas, Texas and all starling samples were processed the day of collection.

External starling samples were collected by washing the starling carcass with $50 \mathrm{~mL}$ of buffered peptone water (BPW; Cole-Palmer Instrument Company, Vernon Hills, IL 60061). Starlings were removed from their Whirl-Paks ${ }^{\circledR}$ and placed in a 1 gallon Ziploc ${ }^{\circledR}$ bag and the $50 \mathrm{~mL}$ of BPW within an autoclaved $125 \mathrm{~mL}$ vial was poured into the bag. All feathers, feet and beak were washed with BPW for approximately $2 \mathrm{~min}$. The BPW wash was poured back into the original $125 \mathrm{~mL}$ vial and immediately packaged for shipping. After BPW wash, birds were dried with $100 \mathrm{~mL}$ absorbent lab mats (American Engineering Fabrics, Inc., New Bedford, MA 02746). Only three starlings had lower GI tracts ruptured with bird shot during collection and none of these external wash samples tested positive for $S$. enterica.

Starling lower GI tracts (duodenum to the cloaca) were removed by cutting vertically from the cloaca through the top of the keel and removing the internal organs. To reduce risk of cross-contamination we cleaned scissors, lab stations and starling abdomens with $70 \%$ ethanol prior each dissection and lab mats and gloves were replaced after every dissection. Lower GI tracts were placed in sterile Whirl-Paks ${ }^{\circledR}$ and immediately packaged for shipping.

\subsection{Sample shipment}

All samples were shipped UPS priority overnight on the day of collection to Colorado State University (CSU), Veterinary Diagnostic Laboratory (VDL) for testing. Samples were packed with Ice-Brix ${ }^{\circledR}$ (Polar Tech Industries, Genoa, IL 60135) and express shipped in insulated boxes for the next delivery day. Only samples received one day after collection were analyzed.

\subsection{Laboratory analysis}

Standard operating procedures for the CSU-VDL were used for Salmonella culture. Briefly, ten-fold dilutions were made of each environmental sample type $(10 \mathrm{~g}$ feed, $25 \mathrm{~mL}$ water, $50 \mathrm{~mL}$ starling wash) in BPW and incubated overnight at $35^{\circ} \mathrm{C}$. After pre-enrichment, $1 \mathrm{~mL}$ of the culture suspension was added to $10 \mathrm{~mL}$ of tetrathionate broth (Difco Laboratories Inc., Detroit, MI 48201) and incubated overnight at $35^{\circ} \mathrm{C}$ (Dargatz et al., 2005). Cattle fecal and starling intestinal samples were added at ten-fold dilutions to tetrathionate broth and incubated overnight at $35^{\circ} \mathrm{C}$ (Kim et al., 2001). For each sample type, $100 \mu \mathrm{L}$ of the incubated tetrathionate suspension was transferred to $10 \mathrm{~mL}$ of Rappaport-Vassiliadis broth (Oxoid, Ogdensburg, NY 13669) and incubated overnight at $42{ }^{\circ} \mathrm{C}$. A swab of the culture suspension was plated for isolation on Brilliant green agar (Difco Laboratories Inc.) and XLT4 agar plates (BD, Franklin Lakes, NJ 07417) and incubated for $24 \mathrm{~h}$ at $35^{\circ} \mathrm{C}$. Up to three suspect colonies based on colony morphology were picked and plated to blood agar plates. Following overnight incubation at $35^{\circ} \mathrm{C}$, colonies were tested with polyvalent O-grouping antisera for agglutination. All positive samples were sent to the USDA/APHIS National Veterinary Services Laboratory (NVSL) in Ames, Iowa for serotyping.

Standard operating procedures for the CSU-VDL were used for antimicrobial susceptibility testing. Isolates were cultured on blood agar plates from frozen bacterial stocks $24 \mathrm{~h}$ before susceptibility testing. Disk-diffusion testing was performed on Müeller-Hinton agar (Difco Laboratories Inc.) and interpreted according to Clinical and Laboratory Standards Institute (CLSI) recommendations (Clinical and Laboratory Standards Institute, 2009) using the Biomic ${ }^{\circledR}$ V3 (Giles Scientific, Santa Barbara, CA 93103). For quality 
control, E. coli (ATCC 25922 and ATCC 35218), S. aureus (ATCC 29213), Enterococcus faecalis (ATCC 29212) and Pseudomonas aeruginosa (ATCC 27853) were used. Susceptibility to 12 antimicrobials was determined; Amikacin (AK), Ampicillin (AMP), Amoxicillin/Clavulanic acid (AMO), Ceftiofur (TIO), Cephalothin (CEP), Chloramphenicol (CHL), Enrofloxacin (ENF), Gentamicin (GEN), Streptomycin(STR), Sulfisoxazole (SUL), Tetracycline (TET), and Trimethoprimsulfamethoxazole (TMS) (Sensi-Discs ${ }^{\text {TM }}$, Cockeysville, MD 21030).

\subsection{Data analysis}

All antimicrobial susceptibility results were classified as susceptible, intermediate, or non-susceptible according to CLSI breakpoints (Clinical and Laboratory Standards Institute, 2009). Resistance phenotypes were characterized for all resistant isolates. A resistance index (RI; number of antimicrobials to which the isolate was not susceptible) was created for all AMR isolates. Isolates were classified as multi-resistant when they were non-susceptible to $\geq 4$ antimicrobials.

Presence of S. enterica was analyzed descriptively for all samples, serogroups and serotypes. Additionally, occurrence of all S. enterica and AMR S. enterica isolates was compared among environmental samples (cattle feed, cattle water and external starling samples) and fecal samples (cattle fecal and starling GI samples) using a Wald Chi-square statistic. Odds ratios were used to measure effect size, which represented the odds of S. enterica being detected in cattle fecal samples versus starling GI samples. Wald Chi-square and odds ratios were calculated using logistic regression in Proc Logistic, SAS 9.2. Analyses of $S$. enterica and AMR S. enterica isolates were conducted separately.

Comparisons between internal and external starling samples for S. enterica and AMR S. enterica were conducted using McNemar's test using Proc Frequency in SAS 9.2. Odds ratios were calculated using logistic regression in Proc Logistic, SAS 9.2. Odds ratios were used to measure

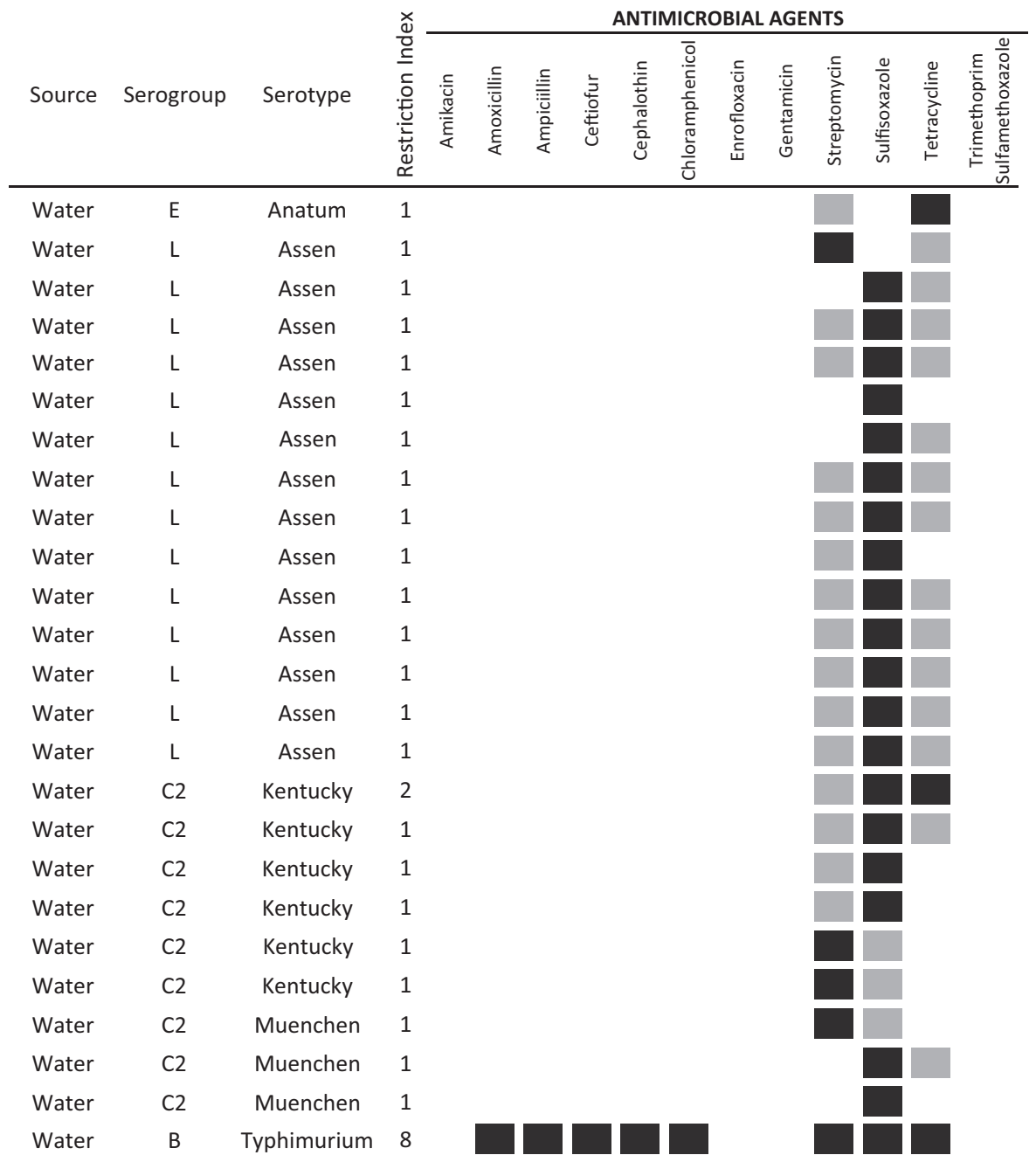

Fig. 1. Antimicrobial resistant phenotypes for Salmonella enterica isolates originating within cattle water trough samples collected from a CAFO in TX, USA, 2012. Isolates were screened for antimicrobial susceptibility. Empty cells denote susceptibility, gray cells denote intermediate resistance, and black cells denote resistance to the antibiotic. Resistance classifications were based upon Clinical and Laboratory Standards Institute 2006 guidelines. 


\begin{tabular}{|c|c|c|c|c|c|c|c|c|c|c|c|c|c|c|c|}
\hline \multirow[b]{2}{*}{ Source } & \multirow[b]{2}{*}{ Serogroup } & \multirow[b]{2}{*}{ Serotype } & \multirow[b]{2}{*}{ 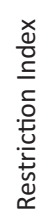 } & \multicolumn{12}{|c|}{ ANTIMICROBIAL AGENTS } \\
\hline & & & & 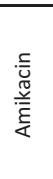 & 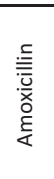 & 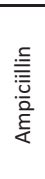 & 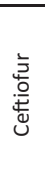 & 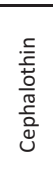 & 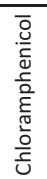 & 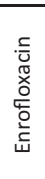 & 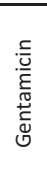 & 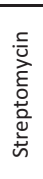 & $\begin{array}{l}\frac{\mathscr{O}}{0} \\
\tilde{N} \\
\text { Oे } \\
\stackrel{\mathscr{C}}{5} \\
\tilde{n}\end{array}$ & 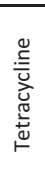 & 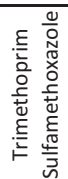 \\
\hline Feed & $\mathrm{C} 2$ & Kentucky & 1 & & & & & & & & & & & & \\
\hline Feed & C2 & Kentucky & 1 & & & & & & & & & & & & \\
\hline Feed & $\mathrm{C} 2$ & Kentucky & 1 & & & & & & & & & & & & \\
\hline Feed & C2 & Kentucky & 1 & & & & & & & & & & & & \\
\hline Feed & C2 & Muenchen & 1 & & & & & & & & & & & & \\
\hline Feed & C2 & Newport & 8 & & & & & & & & & & & & \\
\hline
\end{tabular}

Fig. 2. Antimicrobial resistant phenotypes for Salmonella enterica isolates originating within cattle feed samples collected from a CAFO in TX, USA 2012. Isolates were screened for antimicrobial susceptibility. Empty cells denote susceptibility, gray cells denote intermediate resistance, and black cells denote resistance to the antibiotic. Resistance classifications were based upon Clinical and Laboratory Standards Institute 2006 guidelines.

effect size, which represented the odds of S. enterica being detected in starling GI samples versus external starling wash samples. Analyses of S. enterica and AMR S. enterica isolates were conducted separately.

Comparisons of AMR isolates among serogroups and serotypes were conducted using logistic regression in Proc Logistic, SAS 9.2. The Wald Chi-square statistic was used to assess if differences in occurrence of AMR isolates exist by serogroup among sources (internal starling, external starling, cattle feces, feed and water samples). For the analysis of serotypes, only serotypes with $\geq 10$ isolates were included in the analysis, resulting in a comparison of five serotypes. The Wald Chi-square statistic was used to assess if differences in occurrence of AMR isolates exist among the serotypes assessed.

\section{Results}

Our probability of detecting positive $S$. enterica isolates differed by the type of environmental samples $\left(\chi_{2}{ }^{2}=44.85\right.$, $P<0.0001)$. Among water trough samples, 57\% $(n=100)$ were positive for $S$. enterica and three samples contained two serotypes. Among cattle feed samples, $23 \%(n=100)$ were positive for $S$. enterica. Among external starling wash samples, $17 \%(n=100)$ were positive for S. enterica. Our probability of detecting AMR $S$. enterica isolates also differed by environmental samples $\left(\chi_{2}^{2}=28.82, P<0.0001\right)$. Among all water trough samples, $24 \%$ were positive for AMR $S$. enterica and one was multidrug resistant (Fig. 1). Six percent of all cattle feed samples were positive for AMR S. enterica and one of these isolates was multidrug resistant (Fig. 2). Among all external starling wash samples, $2 \%$ were positive for AMR S. enterica (Fig. 3). Only one reference feed sample (3\%) was positive for S. enterica (Serogroup C1, serotype Montevideo) and it was susceptible to all antibiotics; this sample originated from the mixed rations collected directly from the cattle feed truck.

Our probability of detecting positive $S$. enterica isolates differed between cattle fecal and starling GI samples $\left(\chi_{1}^{2}=9.690, P=0.0019\right)$. Among cattle fecal samples, 54\% $(n=100)$ were positive for S. enterica and one cattle fecal sample contained two serotypes. Among European starling GI samples, $32 \%$ ( $n=100$ ) were positive for S. enterica. Based upon odds ratio analysis the probability of isolating $S$. enterica was greater from cattle fecal samples than it was from starling GI samples $(\mathrm{OR}=2.494,95 \% \mathrm{CI}=1.403,4.435)$. Our probability of detecting AMR S. enterica isolates differed between cattle fecal and starling GI samples $\left(\chi_{1}{ }^{2}=18.487\right.$, $P<0.0001)$. Among all cattle fecal samples, $35 \%(35 / 100)$ were positive for AMR $S$. enterica and none demonstrated multidrug resistance (Fig. 4). Among all European starling GI samples, $8 \%$ were positive for AMR S. enterica and one of these GI samples was multidrug resistant (Fig. 5). Based upon odds ratio analysis the odds of detecting AMR S. enterica was greater from cattle fecal samples than it was from starling GI samples $(\mathrm{OR}=6.192,95 \% \mathrm{CI}=2.697,14.217)$.

Our probability of detecting positive $S$. enterica isolates differed by external and internal European starling

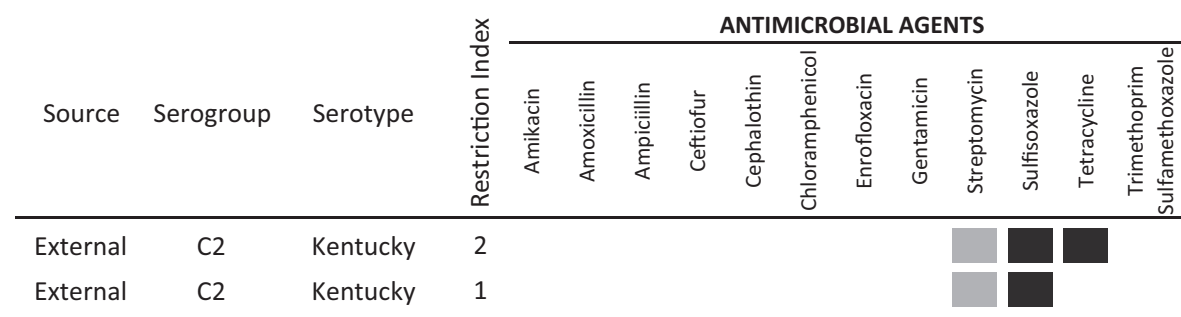

Fig. 3. Antimicrobial resistant phenotypes for Salmonella enterica isolates originating within external starling wash samples collected from a CAFO in TX USA, 2012. Isolates were screened for antimicrobial susceptibility. Empty cells denote susceptibility, gray cells denote intermediate resistance, and black cells denote resistance to the antibiotic. Resistance classifications were based upon Clinical and Laboratory Standards Institute 2006 guidelines. 


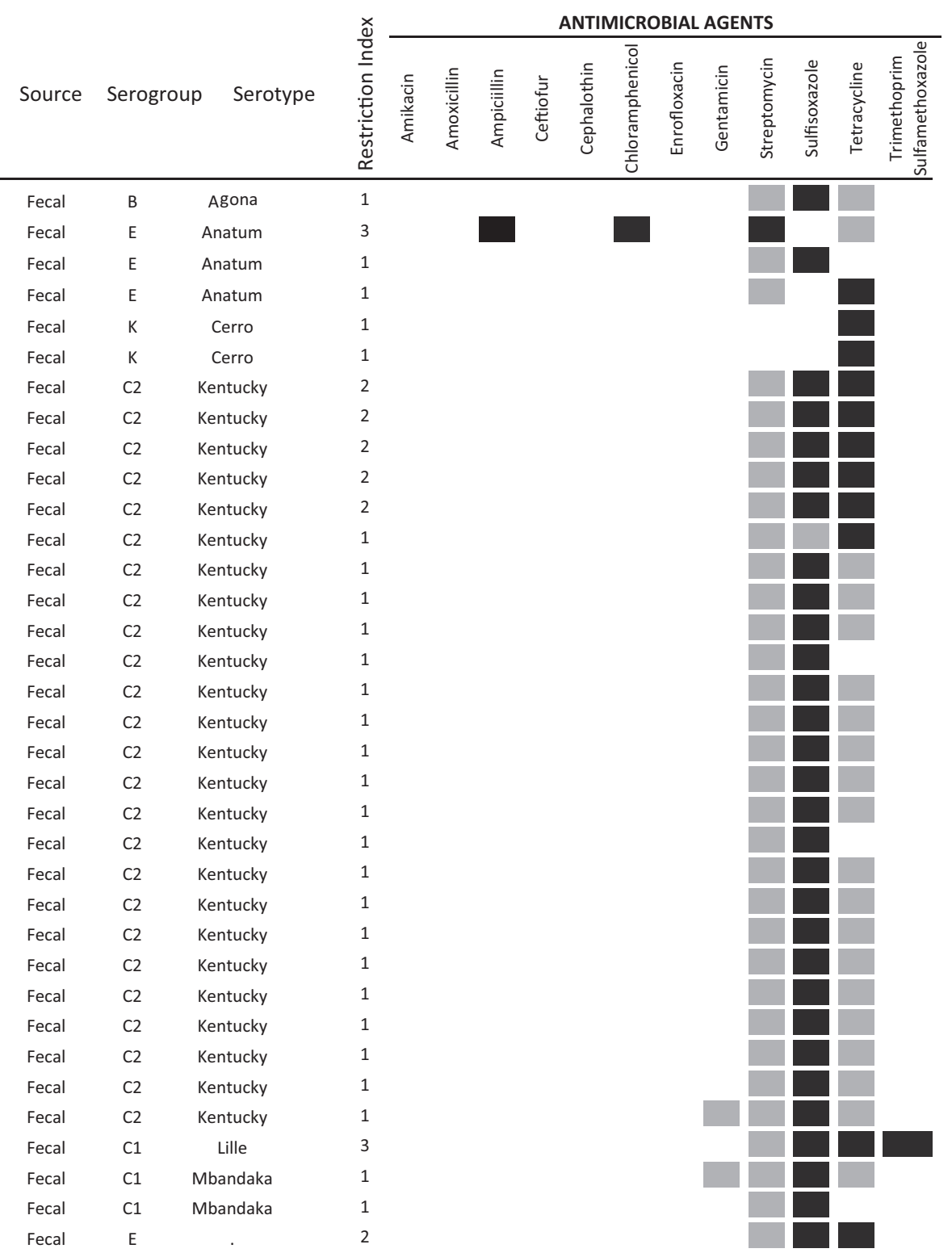

Fig. 4. Antimicrobial resistant phenotypes for Salmonella enterica isolates originating within cattle fecal samples collected from a CAFO in TX, USA, 2012. Isolates were screened for antimicrobial susceptibility. Empty cells denote susceptibility, gray cells denote intermediate resistance, and black cells denote resistance to the antibiotic. Resistance classifications were based upon Clinical and Laboratory Standards Institute 2006 guidelines.

samples $\left(S_{1}=6.429, P=0.011\right)$. Based upon odds ratio analysis, the probability of isolating $S$. enterica is greater from starling GI tract samples than it is from external wash samples (OR $=2.297,95 \% \mathrm{CI}=1.176,4.489$ ). Our probability of detecting AMR $S$. enterica did not differ among external and internal starling samples $\left(S_{1}=3.60\right.$, $P=0.0578$ ). Based on odds ratio analysis, there was no difference in the probability of isolating AMR S. enterica from starling GI tract samples vs external starling wash samples $(\mathrm{OR}=2.500,95 \% \mathrm{CI}=0.467,13.393)$.

Our probability of detecting AMR S. enterica differed by serogroup $\left(\chi_{5}{ }^{2}=32.2987, P<0.0001\right)$ and serotypes (Table 1). Among the five serotypes assessed in our analysis (S. enterica serotypes Kentucky, Anatum, Assen, Montevideo and Muenchen) our probability of detecting antibiotic resistant isolates differed by serotype $\left(\chi_{4}{ }^{2}=28.675, P<0.0001\right)$.

\section{Discussion}

It is important to state that these data do not document transmission from starling to cattle, cattle feed or water, nor do we provide data that the starlings are introducing $S$. enterica to the CAFO from other locations. Data presented in this manuscript do provide proof of concept that starling use of CAFO can lead to the mechanical movement 


\begin{tabular}{|c|c|c|c|c|c|c|c|c|c|c|c|c|c|c|c|}
\hline \multirow[b]{2}{*}{ Source } & \multirow[b]{2}{*}{ Serogroup } & \multirow[b]{2}{*}{ Serotype } & \multirow[b]{2}{*}{ 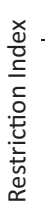 } & \multicolumn{12}{|c|}{ ANTIMICROBIAL AGENTS } \\
\hline & & & & 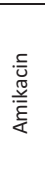 & 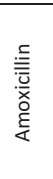 & 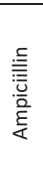 & 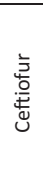 & $\begin{array}{l}\frac{c}{\frac{c}{5}} \\
\frac{0}{\pi} \\
\frac{0}{0} \\
\frac{0}{g}\end{array}$ & 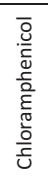 & 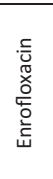 & 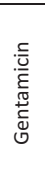 & 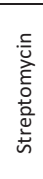 & 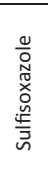 & 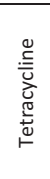 & 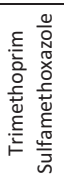 \\
\hline Starling GI & $E$ & Anatum & 1 & & & & & & & & & & & & \\
\hline Starling GI & $\mathrm{E}$ & Anatum & 1 & & & & & & & & & & & & \\
\hline Starling GI & $\mathrm{C} 2$ & Kentucky & 4 & & & & & & & & & & & & \\
\hline Starling GI & $\mathrm{C} 2$ & Kentucky & 2 & & & & & & & & & & & & \\
\hline Starling GI & C2 & Kentucky & 1 & & & & & & & & & & & & \\
\hline Starling GI & B & Reading & 1 & & & & & & & & & & & & \\
\hline Starling GI & $\mathrm{C} 1$ & Mbandaka & 1 & & & & & & & & & & & & \\
\hline Starling GI & $\mathrm{C} 1$ & Mbandaka & 1 & & & & & & & & & & & & \\
\hline
\end{tabular}

Fig. 5. Antimicrobial resistant phenotypes for Salmonella enterica isolates originating within starling gastrointestinal tract samples collected from a CAFO in TX, USA, 2012. Isolates were screened for antimicrobial susceptibility. Empty cells denote susceptibility, gray cells denote intermediate resistance, and black cells denote resistance to the antibiotic. Resistance classifications were based upon Clinical and Laboratory Standards Institute 2006 guidelines.

of $S$. enterica, which provides greater clarity on the mechanisms of microbiological contamination associated with wildlife-livestock interactions. In other words, we documented that foraging flocks of starlings can potentially act as fomites for $S$. enterica within CAFO; an additional PFGE analysis of these and other $S$. enterica isolates recovered from this CAFO supports these findings (Carlson et al., 2015).

Large amounts of fecal material from cattle were present on the exterior of starlings at the time of collection. This fecal material most likely explains why so many external wash samples tested positive for S. enterica. Thus, it is possible that all species of bird using CAFO can mechanically move $S$. enterica to the cattle feed and water supplies they consume. This suggests that starlings, and possibly other birds, do not have to be GI vectors to spread S. enterica within CAFO.
Two external starling wash and eight starling GI samples contained $S$. enterica isolates that were resistant to at least one class of antibiotics and one of the isolates obtained from starling GI samples (S. enterica serotype. Kentucky) was multidrug resistant. Isolating AMR $S$. enterica from starling samples, especially GI samples, suggests that starling-livestock interactions may create a wildlife reservoir for AMR S. enterica.

This is not the first work to implicate starlings as a source for the mechanical movement of microorganisms in CAFO. Coccidia oocysts have been isolated from water sources exclusively used by European starlings livetrapped on CAFO, yet none of the starling GI tract samples tested positive for the coccidia oocyst (Carlson et al., 2011c). This suggested the starlings introduced coccida oocysts to the water through a mechanism other than their own fecal waste.

Table 1

Salmonella enterica serotypes isolated by source. All samples were collected in a concentrated animal feeding operation in TX, USA, January 2012.

\begin{tabular}{|c|c|c|c|c|c|c|c|}
\hline \multirow[t]{2}{*}{ Serotypes } & \multicolumn{7}{|c|}{ Salmonella serotypes } \\
\hline & Serogroup & Internal $^{\mathrm{a}}$ starling & External $^{\mathrm{b}}$ starling & Cattle feed & Cattle water & Cattle feces & Total \\
\hline Agona & B & 1 & 0 & 0 & 2 & 3 & 6 \\
\hline Anatum & $\mathrm{E}$ & 15 & 5 & 5 & 6 & 10 & 41 \\
\hline Anatum_var._15+ & $\mathrm{E}$ & 0 & 0 & 0 & 1 & 0 & 1 \\
\hline Assen & $\mathrm{L}$ & 0 & 0 & 0 & 27 & 0 & 27 \\
\hline Cerro & K & 1 & 0 & 0 & 1 & 2 & 4 \\
\hline Kentucky & $\mathrm{C} 2$ & 3 & 3 & 12 & 8 & 28 & 54 \\
\hline Lillie & $\mathrm{C} 1$ & 0 & 0 & 0 & 0 & 1 & 1 \\
\hline Mbandaka & $\mathrm{C} 1$ & 2 & 0 & 0 & 0 & 2 & 4 \\
\hline Meleagridis & E1 & 1 & 2 & 0 & 2 & 0 & 5 \\
\hline Montevideo & $\mathrm{C} 1$ & 6 & 2 & 1 & 6 & 7 & 22 \\
\hline Muenchen & $\mathrm{C} 2$ & 2 & 4 & 2 & 3 & 1 & 12 \\
\hline Newport & $\mathrm{C} 2$ & 0 & 0 & 1 & 0 & 0 & 1 \\
\hline Reading & $\mathrm{B}$ & 1 & 0 & 0 & 0 & 0 & 1 \\
\hline Rough_0:e,h:1,6 & $\mathrm{E}$ & 0 & 0 & 1 & 1 & 0 & 2 \\
\hline Typhimurium & B & 0 & 0 & 0 & 1 & 0 & 1 \\
\hline Senftenberg & $\mathrm{E}$ & 0 & 0 & 0 & 1 & 0 & 1 \\
\hline Unknown & $\mathrm{C} 2, \mathrm{E}$ & 0 & 1 & 1 & 1 & 1 & 4 \\
\hline Total & & 32 & 17 & 23 & 60 & 55 & 187 \\
\hline
\end{tabular}

a Internal starling samples refer to lower gastrointestinal tracts, duodenum to the cloaca, that were removed for laboratory analysis.

b External starling samples refer to $50 \mathrm{~mL}$ buffered water samples that were used to wash the outside of each starling collected for laboratory analysis. 
There were ecologically important differences in $S$. enterica occupancy within the CAFO environment. Serogroup C2 was the most common serogroup detected within the CAFO, comprising $37.9 \%$ of all the isolates recovered; it was also the most commonly associated serogroup with cattle fecal samples $40.8 \%$. Serogroup E was the second most common serogroup detected. It comprised $27.2 \%$ of all the isolates recovered and it was most common within starling GI tract samples $31.3 \%$. Serogroup $\mathrm{L}$ was only detected within cattle water troughs and it comprised $14.4 \%$ of all isolates detected. These data suggests diversity and maintenance of S. enterica in CAFO is strongly influenced by the interactions between serogroup, animal, and the environmental media being contaminated (feed, water, animal hide, equipment, and so on). With a better understanding of $S$. enterica microbial ecology in CAFO, targeted and cost-effective management actions could potentially be implemented to reduce the diversity and quantity of $S$. enterica.

Serogroup C2 contained the most AMR S. enterica isolates with $61.9 \%$ exhibiting resistance to one or more antibiotics and the majority of multidrug resistant isolates $66 \%$. Among serogroup L, $48.1 \%$ of isolates were resistant to one or more antibiotics. Serogroup E contained the lowest percentage of AMR isolates, only $15.6 \%$ were resistant to one or more antibiotics. Differences in occurrence of AMR isolates by serogroups suggest acquisition of Salmonella AMR genes may be influenced by somatic antigens. Since serogroups differed by animal and environmental sources, a more comprehensive understanding of $S$. enterica ecology in CAFO may reveal targeted cleaning and control options that can reduce AMR S. enterica fecal shedding by cattle. For example, water troughs compared to other environmental sources harbored a disproportionally large number of AMR $S$. enterica isolates. Increased cleaning frequencies, or novel trough designs that reduce microbial contamination may reduce occurrence of AMR S. enterica isolates in CAFO.

Important differences appear to exist among AMR $S$. enterica serotypes. S. enterica serotype Kentucky contained the greatest number of resistant isolates $(74.1 \%)$, the majority of these were isolated from cattle fecal samples (62.5\%) and S. enterica serotype Kentucky accounted for the majority of AMR isolates found within serogroup C2 (90.9\%). Additionally, one AMR isolate, S. enterica serotype Kentucky, was multidrug resistant and it was recovered from a starling GI tract. S. enterica serotype Assen also contained a large number of antibiotic resistant isolates (48.1\%). It was the only isolate detected in serogroup L and it was found exclusively in cattle water troughs. Among all sample types, cattle fecal samples contained the most AMR isolates, suggesting that cattle were the primary source for AMR S. enterica within the CAFO. Yet, water troughs and starlings contributed some unique AMR isolates which suggests water troughs and wildlife can contribute to the persistence and environmental dissemination of AMR $S$. enterica isolates in CAFO.

Starling ecology suggests there may be public health risks associated with their use of CAFO. Starlings exploit CAFO in winter for food resources and leave in spring when insects become abundant (Linz et al., 2007). During the spring and summer these birds are commonly found breeding in suburban and urban environments (Blair, 1996; Melles et al., 2003). When considered in this broader context, our data suggests starlings may acquire $S$. enterica during the winter then migrate to residential and urban landscapes to breed. Their unique ecology suggests starlings may be a high risk species for the spread pathogenic bacteria and AMR genes within environments that can create public health risks. To better understand if public health risks are created as a consequence of starlinglivestock interactions experimental infection studies with starlings should be conducted to determine severity and duration of $S$. enterica fecal shedding.

\section{Conflict of interests}

We have no conflict of interests to report.

\section{Acknowledgements}

This project was funded through the USDA/APHIS/ National Wildlife Disease Program. Guidance and technical assistance was provided by Texas Cattle Feeders Association, Texas Wildlife Services and the National Wildlife Research Center.

\section{References}

Armstrong, G.L., Hollingsworth, J., Morris, J.G., 1996. Emerging foodborne pathogens: Escherichia coli $0157: \mathrm{H} 7$ as a model of entry of a new pathogen into the food supply of the developed world. Epidemiol. Rev. 18, 29-51.

Blair, R.B., 1996. Land use and avian species diversity along an urban gradient. Ecol. Appl. 6, 506-519.

Brichta-Harhay, D.M., Arthur, T.M., Bosilevac, J.M., Kalchayanand, N., Shackelford, S.D., Wheeler, T.L., Koohmaraie, M., 2011. Diversity of multidrug-resistant Salmonella enterica strains associated with cattle at harvest in the United States. Appl. Environ. Microbiol. 77, 1783-1796.

Carlson, J.C., Franklin, A.B., Hyatt, D.R., Pettit, S.E., Linz, G.M., 2011a. The role of starlings in the spread of Salmonella within concentrated animal feeding operations. J. Appl. Ecol. 2, 479-486.

Carlson, J.C., Engeman, R.M., Hyatt, D.R., Gilliland, R.L., DeLiberto, T.J., Clark, L., Bodenchuk, M.J., Linz, G.M., 2011b. Efficacy of European starling control to reduce Salmonella enterica contamination in a concentrated animal feeding operation in the Texas panhandle. BMC Vet. Res. 7, 9.

Carlson, J.C., Linz, G.M., Ballweber, L.R., Elmore, S.A., Pettit, S.E., Franklin, A.B., 2011c. The role of European starlings in the spread of coccidia within concentrated animal feeding operations. Vet. Parasitol. 180, 340-343.

Carlson, J.C., Hyatt, D.R., Bentler, K., Mangan, A.M., Russell, M., Piaggio, A.J., Linz, G.M., 2015. Molecular characterization of Salmonella enterica isolates associated with starling-livestock interactions. Vet. Microbiol., http://dx.doi.org/10.1016/j.vetmic.2015.03.015.

Cernicchiaro, N., Pearl, D.L., McEwan, S.A., Harpster, L., Homan, H.J., Linz, G.M., LeJeune, J.T., 2012. Association of wild bird density and farm management factors with the prevalence of $E$. coli 0157 in dairy herds in Ohio (2007-2009). Zoonoses Public Health 59, 320-329.

Clinical and Laboratory Standards Institute, 2009. Performance Standards for Antimicrobial Disk and Dilution Susceptibility Tests for Bacteria Isolated from Animals: Approved Standard, M31-A3. vol. 28(8), third ed..

Dargatz, D.A., Strohmeyer, R.A., Morley, P.S., Hyatt, D.R., Salman, M.D., 2005. Characterization of Escherichia coli and Salmonella enterica from cattle feed ingredients. Foodborne Pathog. Dis. 2, 341-347.

Depenbusch, B.E., Drouillard, J.S., Lee, C.D., 2011. Feed depredation by European starlings in a Kansas Feedlot. Hum. Wildl. Interact. 5, 58-65.

Dolbeer, R.A., Woronecki, P.P., Stickley, A.R., White, S.B., 1978. Agricultural impacts of a winter population of blackbirds and starlings. Wilson Bull. 90, 31-44. 
Fedorka-Cray, P.J., Dargatz, D.A., Thomas, L.A., Gray, J.T., 1998. Survey of Salmonella serotypes in feedlot cattle. J. Food Prot. 61, 525-530.

Gaukler, S.M., Linz, G.M., Sherwood, J.S., Dyer, N.W., Bleier, W.J., Wannemuehler, Y.M., Nolan, L.K., Logue, C.M., 2009. Escherichia coli, Salmonella, and Mycobacterium avium subsp. paratuberculosis in Wild European Starlings at a Kansas Feedlot. J. Avian Dis. 53, 544-551.

Kauffman, M.D., LeJeune, J., 2011. European starlings (Sturnus vulgaris) challenged with Escherichia coli 0157 can carry and transmit the human pathogen to cattle. Appl. Microbiol. 53, 569-601.

Kim, L.M., Morley, P.S., Traub-Dargatz, J.L., Salman, M.D., Gentry-Weeks, C., 2001. Factors associated with Salmonella shedding among equine colic patients at a veterinary teaching hospital. J. Am. Vet. Med. Assoc. 218, 740-748.

Kirk, J.H., Holmberg, C.A., Jeffrey, J.S., 2002. Prevalence of Salmonella spp in selected birds captured on California dairies. J. Am. Vet. Med. Assoc. 220, 359-362.

Linz, G.M., Homan, H.J., Gaukler, S.M., Penry, L.B., Bleier, W.J., 2007. European starlings: a review of an invasive species with far-reaching impacts. In: Witmer, G.W., Pitt, W.C., Fagerstone, K.A. (Eds.), Managing Vertebrate Invasive Species: Proceedings of an International Symposium. USDA/APHIS/WS, National Wildlife Research Center, Fort Collins, pp. 378-386.

Loo, I.V., Huijsdens, X., Tiemersma, E., Neeling, A.D., Sande-Bruinsma, N.V. Beaujean, D., Voss, A., Kluytmans, J., 2007. Emergence of methicillinresistant Staphylococcus aureus of animal origin in humans. Emerg. Infect. Dis. 13, 1834-1839.

Melles, S., Glenn, S., Martin, K., 2003. Urban bird diversity and landscape complexity: species-environment association along a multiscale habitat gradient. Conserv. Ecol. 7, 5.

Pedersen, K., Clark, L., Andelt, W.F., Salman, M.D., 2006. Prevalence of shiga toxin-producing Escherica coli and Salmonella enterica in rock pigeons captured in Fort Collins, Colorado. J. Wildl. Dis. 42, 46-55.

Shwiff, S.A., Carlson, J.C., Glass, J.H., Suckow, J., Lowney, M.S., Moxcey, K.M., Larson, B., Linz, G.M., 2012. Producer survey of bird-livestock interactions in commercial dairies. J. Dairy Sci. 95, 6820-6829. 